\title{
APLICACIONES DE LA FÍSICA ESTADÍSTICA EN LA VALORACIÓN DE ACTIVOS FINANCIEROS: DE LA ECUACIÓN DE FOKKER-PLANCK AL MODELO DE BLACK-SCHOLES. SOLUCIÓN EN DIFERENCIAS FINITAS PARA UNA OPCIÓN PUT EUROPEA.
}

JOSÉ RAFAEL CARO BARRERA

Dpto. de Estadística, Econometría, Investigación Operativa y Métodos Cuantitativos. UNIVERSIDAD DE CÓRDOBA, ESPAÑA

E-mail: z52cabaj@uco.es

\section{RESUMEN}

Las técnicas estocásticas y probabilísticas juegan un papel fundamental en la modelización matemática de aspectos relacionados con las ciencias naturales y sociales. En física, los modelos estocásticos son usados con frecuencia en áreas tan diversas como la climatología, la biología molecular, la bioquímica, así como en la economía. El propósito de este trabajo es el de plantear la aplicación a las finanzas de modelos y procesos utilizados en el campo de la física estadística, mediante técnicas y resultados de la teoría estocástica de procesos y en particular de procesos de difusión que, directamente surgidos y aplicados en el campo de la física tienen su utilidad en el campo de la economía financiera. En nuestro caso, se pretende relacionar la ecuación de Fokker-Planck con el modelo planteado por Black y Scholes dado que éste último es modelizado mediante una ecuación parcial diferencial estocástica y pueden establecerse similitudes con los procesos estocásticos y de difusión que se observan en la física.

Palabras clave: Física Estadística, Física Mecánica, Econofísica, Procesos Estocásticos, Métodos Computacionales, Derivados Financieros, Elementos Finitos, Proceso de Difusión.

Statistical Physics Applications for the Valuation of Financial Assets: From the FokkerPlanck Equation to the Black-Scholes Model. Finite Difference Solution for European Put Options.

\section{ABSTRACT}

The stochastic and probabilistic techniques play a fundamental role in the mathematical modeling of aspects related to the natural and social sciences. In physics, stochastic models are often used in areas as diverse as climatology, molecular biology, biochemistry, as well as economics. The purpose of this paper is to propose the application to finance of models and processes used in the field of statistical physics, through techniques and results of the stochastic theory of processes and in particular of diffusion processes that, directly emerged and applied in the field of physics are useful in the field of financial economics. In our case, we intend to relate the Fokker-Planck equation to the model proposed by Black and Scholes, since the latter is modeled by a stochastic differential partial equation and similarities can be established with the stochastic and diffusion processes observed in physics.

Keywords: Statistical Physics, Mechanical Physics, Econophysics, Stochastic Processes, Computational Methods, Financial Derivatives, Finite Elements, Diffusion Process.

Clasificación JEL: A12, B16, C02, C32, C62, C63, D53, G12

Artículo recibido el 16 de febrero de 2019 y aceptado el 24 de mayo de 2019

Artículo disponible en versión electrónica en la página www.revista-eea.net

ISSN 1697-5731 (online) - ISSN 1133-3197 (print) 


\section{FÍSICA, FINANZAS Y MÉTODOS NUMÉRICOS. UNA BREVE INTRODUCCIÓN A LA ECONOFÍSICA.}

Aunque precursores de la economía moderna tales como Bachelier, que argumentó que las fluctuaciones del precio se comportaban como un camino aleatorio y las modelizó de acuerdo a un movimiento browniano; Walras en su "Teoría del Equilibrio General"; Pareto y su distribución de la riqueza o incluso más reciente, el económetra Georgescu-Roegen, que considera la ley de la entropía como la más afín al proceso económico (Georgescu-Rogen, 1971), han formulado y aplicado métodos de la física en la economía, estas dos disciplinas no han estado unidas a lo largo de la historia.

En palabras de Roehner (2002), quizás, el primer pre-econofísico fue Quételet cuando estableció un método para comparar tanto la física como los datos sociales y, aunque durante toda su vida fue astrónomo, tuvo un fuerte interés personal en las ciencias sociales y en particular, en cuestiones demográficas.

Según Schinckus (2010), desde hace algún tiempo ha empezado a haber cierta conexión entre la física y la economía, surgiendo, según Gingras y Schinckus (2012) "una disciplina que puede ser considerada como una nueva perspectiva de la economía." De este modo, no se puede hablar de "econofísica" como tal hasta hace bien poco dado que el término no se usó por primera vez hasta 1995, concretamente en una conferencia internacional sobre física estadística en Kolkata (India) por el Dr. H. Eugene Stanley. Es, por tanto, en esos años de mitad de la década de los 90 cuando algunas revistas del campo de la física empiezan a publicar en sus contenidos artículos con estudios económicos. Desde entonces, los físicos se han ido involucrando cada vez más en el análisis de los sistemas económicos y financieros desde su óptica y con hipótesis, planteamientos y modelos de su campo, alegando que los procedimientos son más adecuados que los que disciplinas tradicionales relacionadas con los hechos económicos venían planteando.

\subsection{Contribución de la econofísica a la economía}

Podríamos clasificar en cuatro las áreas de la economía en las que la econofísica ha contribuido, y está contribuyendo, de forma activa y a la luz de la ingente literatura publicada, estas son:

Mercados financieros,

1) Distribución de la riqueza e ingresos,

2) Economía industrial (distribución del tamaño de las compañías y tasas de crecimiento) y

3) Análisis de redes,

ya que existe una creencia casi común entre los "econofísicos" de que las regularidades empíricas universales pueden descubrirse al menos en estas áreas (Kutner y Grech, 2008). Según estos autores, todos los estudios en econo- y sociofísica pueden clasificarse en tres categorías, independientemente de si se basan en:

(i) métodos estadísticos, incluida la termodinámica fenomenológica de los estados de equilibrio y no equilibrio o la física estadística exótica (o no gaussiana y no exponencial),

(ii) métodos deterministas; esta categoría se basa, por ejemplo, en teorías de campo o la teoría del caos determinista, $\mathrm{y}$

(iii) métodos híbridos, donde los enfoques deterministas son perturbados por diferentes ruidos estocásticos (la dinámica de tipo Langevin, que se suministra con un ruido blanco, es aquí un ejemplo de referencia).

En este sentido, haciendo una sucinta revisión de la literatura, diversos autores han tomado prestados modelos de la física y aplicado a los más diversos campos de la economía; por ejemplo, Ilinski (2001) aplica conceptos de la electrodinámica a la dinámica de mercados en situación de no-equilibrio; Ingber (1990) usa elementos de la mecánica estadística para modelizar la estructura de tipos de interés; Watts (2004) emplea la teoría de grafos para explicar cómo todo está conectado; Schanden (2002) utiliza la teoría cuántica para modelizar mercados financieros secundarios y Voit, en su obra The Statistical Mechanics of Financial Markets, da una interesante introducción sobre las propiedades estadísticas de los mercados financieros para los físicos, y entre otras aplicaciones, demuestra que los movimientos de precios en escalas de tiempo que varían en varios órdenes de magnitud pueden colapsarse en una curva 
universal mediante una simple reescala del intervalo de tiempo y la densidad de probabilidad observada (Voit, 2005).

Más recientemente, Baaquie (2013), emplea elementos de la matemática cuántica para describir la evolución aleatoria de instrumentos financieros; Herzog (2015), va más allá e incluso se atreve a proponer un modelo econofísico para las burbujas financieras; en cambio, James Chen, usa elementos de la física subatómica y la física de partículas para separar el riesgo sistemático, recogido en el parámetro beta del modelo de valoración de activos financieros (modelo CAPM ${ }^{1}$ en inglés) (Chen, 2018) y aquí en España, por ejemplo, Trinidad Segovia y otros (2018), han establecido, recientemente, paralelismos entre la dinámica que desarrollan las partículas de diferentes materiales con la evolución de los mercados financieros, concretamente con el mercado de cambio de divisas.

Todos estos autores, la mayoría físicos o muy ligados a este campo por la naturaleza de sus investigaciones, por citar algunos, han visto posible utilizar esos modelos porque pueden reflejar ciertas características de las finanzas cuantitativas modernas y piensan que se debe buscar un nuevo enfoque más comparativo y basado en evidencias empíricas más "robustas" que las que proporcionaban, hasta ahora los métodos tradicionales.

Sin embargo, debemos hacer algunas observaciones, ya que tal y como señalan Jovanovic y Schinckus (2010), han surgido discusiones entre físicos y economistas que no logran ver la unión entre ambos campos. Así, estos autores han analizado los principales orígenes de estas dificultades en orden a contribuir al desarrollo de puentes metodológicos y teóricos entre estas dos disciplinas, estudiando las razones por las que ciertos economistas son reacios a la aplicación de la física estadística a las finanzas y exponiendo, básicamente, tres argumentos: 1) la econofísica es un campo orientado a los datos sin fundamentos teóricos, 2) esta área no puede contribuir, de modo real, a la teoría financiera existente y 3) el marco de referencia de la economía financiera no está lo suficientemente considerado por la física.

Por otro lado, el uso de métodos numéricos para la resolución de problemas en el ámbito de las finanzas cuantitativas está ampliamente desarrollado para dar respuestas a la mayoría de cuestiones planteadas en un campo tan vasto como el que nos ocupa. Algunos de estos problemas son de fácil resolución, o al menos no plantean excesivas dificultades, pudiendo ser solucionados en su forma más básica mediante métodos numéricos estándar. Sin embargo, de acuerdo con Brandimarte, se necesitan métodos y herramientas más sofisticadas cuando surgen limitaciones a la formulación básica de ciertos problemas (Brandimarte, 2002). Es decir, además del uso de métodos numéricos tales como programación matemática (incluyendo programación estocástica y de optimización ${ }^{2}$ ), métodos de simulación Monte Carlo y métodos en diferencias finitas para resolver ecuaciones diferenciales parciales, se antoja necesario el uso de software adecuado a tales fines.

Los mercados financieros son sistemas dinámicos complejos y en continua evolución. Para dar respuesta a esta complejidad y predecir esta evolución, los economistas, tradicionalmente, han elaborado modelos de simulación basados en las técnicas clásicas, como la mencionada anteriormente simulación Monte Carlo, con herramientas que proporcionaban la estadística y la econometría. Estos modelos clásicos, consideraban que los mercados estaban formados por agentes homogéneos, con preferencias y características iguales y constantes a lo largo del tiempo, que en caso de comunicarse lo hacían con interacciones muy simples, donde además se suponía que el sistema subyacente se mantenía en equilibrio.

Sin embargo, cuando se observa la realidad, concretamente la realidad financiera, se hace evidente que estas premisas de partida están lejos de lo que realmente sucede en los mercados financieros, ya que esa "aparente" homogeneidad de los agentes en los mercados se torna heterogeneidad, pues poseen preferencias y características desiguales y no lineales (o no constantes) a lo largo del tiempo (Pavard y Dugdale, 2001), lo que provoca un desequilibrio constante, que pone de manifiesto la "imperfección" de los mercados, haciendo que la hipótesis del mercado eficiente sea puesta en duda, como así se encargaron de demostrar Akerlof, Stiglitz y Spence, cuyas investigaciones les otorgaron el Premio Nobel en 2001.

\footnotetext{
1 'CAPM', en sus siglas en inglés para 'Capital Asset Pricing Model'.

${ }^{2}$ Mixed integer programming (MIP) o programación en enteros.
} 
Una aproximación muy interesante sobre el tema de los mercados financieros y con un tratamiento más amplio, desde el punto de vista de la econofísica, sobre el tema del escalado y las correlaciones en los mismos, se pueden encontrar en Mantegna y Stanley (2000). En esa línea, Borland, Bouchaud y otros (2006) introducen la idea 'multifractal', , relacionada con el escalado para modelizar las principales características estadísticas de las series cronológicas financieras, señalando algunas deficiencias de los modelos actuales, en particular con respecto a la simetría de inversión de tiempo, y proponen una familia alternativa de modelos de múltiples escalas temporales, intermedias entre los modelos GARCH y los modelos multifractales, con resultados prometedores y Edgar (1994) aplica la teoría del caos a la economía y la inversión en los mercados financieros, por poner algunos ejemplos.

\subsection{Motivación y Estructura.}

El objetivo que nos proponemos en los siguientes apartados es el de desarrollar un método determinista para la valoración de opciones financieras, concretamente, la valoración de una opción europea que "promete" una rentabilidad $f\left(S_{T}\right)$ en tiempo T con una función de retorno (o payoff) $f: f(S)=(K-S)_{+}$. Para este cálculo, adoptamos el modelo de Black-Scholes, donde el subyacente evoluciona de acuerdo con la ecuación diferencial estocástica

$$
d S_{t}=S_{t}\left(r d t+\sigma d W_{t}\right), \quad S_{0}=S
$$

donde $S$ denota el precio spot (actual) del subyacente activo con riesgo.

De este modo, el precio justo de una opción put simple (plain Vanilla) ${ }^{4}$ con un retorno $f$ y un vencimiento $\mathrm{T}$, expresado de la forma

$$
V(S, 0)=\mathbb{E}\left[e^{-r T} f\left(S_{T}\right)\right]
$$

y basado en el hecho de que las cantidades como $\mathrm{V}(\mathrm{S}, \mathrm{t})$ se pueden caracterizar como soluciones de ecuaciones diferenciales parciales deterministas (EDP), partiendo o aplicando, por ejemplo, la ecuación diferencial estocástica de Fokker Planck.

De este modo, en la sección 2, se revisa la ecuación Fokker-Planck al mismo tiempo que se establece la relación con nuestro problema financiero propuesto de la valoración de activos; en la sección 3 se plantean las hipótesis de partida y se define la estructura y dimensión del problema; en la sección 4 se aplica el correspondiente método computacional con el software Matlab $\Subset$ para investigar la precisión y el comportamiento bajo diferentes condiciones de contorno y/o para diferentes discretizaciones de parámetros. Finalmente, en la última sección, se expondrán las conclusiones obtenidas y se establecerán posibles líneas de trabajo.

\section{LA ECUACIÓN FOKKER-PLANCK Y LA CONEXIÓN ENTRE LA FÍSICA Y LAS FINANZAS.}

En 1913, en su tesis doctoral, Adrian Fokker, plantea la ecuación y Max Planck la deriva y la desarrolla como una ecuación diferencial de segundo orden. Esta ecuación describe la evolución temporal de la densidad de probabilidad de la partícula browniana en un fluido (Risken y Hannes, 1996) $\mathrm{y}$ no tiene una única solución dado que contiene variables aleatorias y describe, no solo la estacionariedad, sino también la dinámica del sistema, si se utiliza la solución dependiente del tiempo apropiada.

La ecuación de Fokker-Planck es una ecuación de difusión temporal hacia adelante ${ }^{5}$ que describe un proceso de Markov como convención/deriva combinada con difusión:

\footnotetext{
${ }^{3}$ Benoît Mandelbrot fue el primero en introducir el concepto de geometría fractal en las matemáticas, cuyo concepto va más allá que la geometría convencional pues trata de explicar que la mayoría de los sistemas físicos de la naturaleza y muchos artefactos humanos no son formas geométricas regulares (conceptos de la teoría Euclidiana clásica).

${ }^{4}$ Los contratos de opciones más simples son los denominados, en la nomenclatura anglosajona "European Vanilla".

${ }^{5}$ Del inglés 'forward equation', que junto a las ecuaciones retroactivas ( 'backward equations') por lo general, se refieren a las ecuaciones diferenciales que rigen la función de densidad de probabilidad de transición para un proceso estocástico. Son ecuaciones de difusión y, por lo tanto, deben resolverse en la dirección apropiada en el tiempo, de ahí los nombres.
} 


$$
\frac{\partial}{\partial t} P(x, v, t)=-v \frac{\partial}{\partial x} P(x, v, t)-\frac{\partial}{\partial v}\left[\left(\frac{\gamma}{m} v-\frac{1}{m} F(x)\right) P(x, v, t)\right]+\frac{g}{2 m^{2}} \frac{\partial^{2}}{\partial v^{2}} P(x, v, t)
$$

No es el propósito de este artículo la derivación completa de la ecuación anterior, baste decir que (2) es la ecuación de Fokker-Planck para la probabilidad $P d x d v$ de encontrar la partícula Browniana en el intervalo $(x, x+d x) y(v, v+d v)$ en el tiempo t.

De hecho, el movimiento browniano sigue esta última ecuación, aunque, como alternativa puede utilizarse la ecuación Fokker-Planck considerando una densidad de probabilidad en la velocidad y el tiempo $f(v, t)$.

En física, por ejemplo, la interacción de ondas de radio-frecuencia con plasma es descrita por una ecuación Fokker-Planck con un término añadido casi lineal (Stephen y Yau, 2004). Otros autores (Desloge, 1963), han aproximado con la ecuación Fokker-Planck al término de interacción en la ecuación de transporte de Boltzmann con aplicaciones y resultados para gases completamente ionizados. Por otro lado, Ligou (Ligou, 2006) propone una derivación de la ecuación Boltzmann-Fokker-Planck desarrollada en el marco de la teoría cinética del gas.

En nuestro caso, nos proponemos hacer eso mismo que han hecho los autores citados anteriormente, pero aplicándolo, como se ha mencionado con anterioridad, a la valoración de opciones, modelo más conocido como Black-Scholes.

\subsection{El modelo de Black-Scholes.}

En 1973 Fisher Black y Myron Scholes plantearon un modelo para valorar derivados financieros, concretamente opciones, basado en la hipótesis (ya planteada por Bachelier) de que el logaritmo de las fluctuaciones de precio sigue un movimiento browniano; de hecho, su célebre y ampliamente conocida ecuación, bautizada como ecuación de Black-Scholes y galadornada (como resultado de sus investigaciones) con el premio Nobel de Economía en 1997 (Black y Scholes, 1973), no deja de ser una ecuación diferencial. El ambiente de incertidumbre de la valoración del precio de estas opciones está basado en un sistema con dos posibles estados; sin embargo, el poder predictivo de los modelos de valoración numéricos está limitado por varias fuentes de error. La primera de estas fuentes de error es el llamado error de modelización y es debido a las siguientes (y posiblemente 'irrealistas') premisas sobre las cuales se basa la teoría de Black y Scholes:

a) No hay costes de transacción (hipótesis de mercados sin fricción).

b) Ausencia de arbitraje (hipótesis de mercado eficiente).

c) El proceso de valoración St es un movimiento Browniano geométrico.

d) La tasa sin riesgo, $\mathrm{r}$, y la volatilidad $\sigma$ son constantes para $0 \leq t \leq T$

Dejando a un lado el anteriormente mencionado error de modelización, nos situamos en un mercado que se asume sigue un proceso Black y Scholes en el que el precio spot del activo con riesgo es modelizado por un movimiento browniano St con la siguiente ecuación diferencial estocástica

$$
d S_{t}=S_{t}\left(r d t+\sigma d W_{t}\right), \quad S_{0}=S,
$$

donde en aras de la simplicidad se asume que no se pagan dividendos y que se trata de una opción Europea $^{6}$. En base pues, a todo lo anterior, la ecuación de Fokker-Planck aplicada a la anterior ecuación diferencial estocástica (3) es la mencionada ecuación de Black-Scholes (en adelante BS).

Basándonos en el teorema de Feynman-Kac, que establece la unión entre las ecuaciones parciales diferenciales parabólicas y los procesos estocásticos (Simon, 1979), obtenemos momentos como el precio descontado de un contrato de opción europeo con payoff $\mathrm{f}(\mathrm{S})$, es decir, $\mathbb{E}\left(\exp (r-T) f\left(S_{t}\right)\right)$ es igual a la solución $\mathrm{V}(\mathrm{S}, 0)$ de la ecuación Black-Scholes, (en adelante BS).

$$
\frac{\partial V}{\partial t}+\frac{\sigma^{2}}{2} S^{2} \frac{\partial^{2} V}{\partial S^{2}}+r S \frac{\partial V}{\partial S}-r V=0 \quad \text { en } \quad D
$$

en el dominio

\footnotetext{
${ }^{6}$ Una opción europea es que aquella que solo puede ser ejercida al final, es decir, en su madurez.
} 


$$
D=\{(S, t): S>0, \quad 0 \leq t \leq T\}
$$

Así, la ecuación de BS (4) ha de ser completada con la condición terminal

$$
V(S, T)=\text { rentabilidad (payoff) }
$$

la cual depende del tipo de opción. $V$, asimismo, también debe satisfacer unas condiciones de contorno ${ }^{7}$ en $S=0$ y $S \rightarrow \infty$. Por ejemplo, para una opción call:

$$
V(0, t)=0, \quad V(S, t) \sim S-K e^{-r(T-t)} \quad S \rightarrow \infty, 0<t<T
$$

Así, la ecuación BS (4) es una ecuación parcial diferencial parabólica con el operador diferencial "espacial" de segundo orden

$$
A^{B S}=\frac{\sigma^{2}}{2} S^{2} \frac{\partial^{2}}{\partial S^{2}}+r S \frac{\partial}{\partial S}
$$

lo que degenera en $S=0$. Téngase en cuenta que $A^{B S}$ es de tipo Euler, es decir, los coeficientes del polinomio (en $S$ ) tienen la misma homogeneidad que el orden de la diferenciación.

La ecuación BS (4) se puede resolver analíticamente siendo " $\sigma$ " y " $r$ " constantes y para los derivados europeos más simples. Sin embargo, para derivados más complejos, tales como opciones americanas (American contracts), opciones dependientes del recorrido (path-dependent options), etc., no es posible su resolución mediante técnicas analíticas, siendo necesarios métodos numéricos. En el caso que nos ocupa nos hemos centrado en el caso más simple, así en los puntos siguientes, desarrollamos: por un lado, el aparato matemático que nos permite plantear el problema para, a continuación, aplicar los correspondientes métodos que arrojarán como resultado la solución óptima bajo diferentes condiciones límite y/o diferentes parámetros de discretización.

En línea con Courant y otros (1928), problemas de la física matemática clásica que incluyan ecuaciones diferenciales parciales pueden ser reducidos, con objeto de simplificar su estructura, reemplazando los diferenciales por cocientes de diferencia en alguna (rectilínea, por ejemplo) malla, de hecho, su trabajo desarrolla una solución para estos problemas algebraicos, en particular del comportamiento de la solución cuando el ancho de malla tiende a cero.

De forma similar y en nuestro caso, en el desarrollo de los métodos numéricos, así como de la formulación de la solución exacta de la ecuación BS, resulta ciertamente ventajoso transformar dicha ecuación en una forma más simple y que permitan diseñar algoritmos con un rendimiento independiente de los parámetros en el modelo BS; por ello, la reduciremos, previamente a su forma estándar e impondremos unas condiciones de contorno.

\subsection{La ecuación Black-Scholes en log-precio y la configuración de un dominio acotado.}

Téngase en cuenta que el operador diferencial ABS degenera cerca de $S=0$, de modo que para obtener un operador que no se comporte de ese modo tenemos que transformar el proceso del precio $\mathrm{Xt}=\log (\mathrm{St})$, el cual resuelve la ecuación diferencial estocástica

$$
d X_{t}=\left(r-\frac{\sigma^{2}}{2}\right) d t+\sigma d W_{t}
$$

El generador infinitesimal para este proceso tiene coeficientes constantes y es dado por

$$
A^{B S-\log }=\frac{\sigma^{2}}{2} \frac{\partial^{2}}{\partial x^{2}}+\left(r-\frac{\sigma^{2}}{2}\right) \frac{\partial}{\partial x} .
$$

Estableciendo

$$
V(S, t)=v(\log S, T-t)
$$

y obteniendo que $v(x, \tau)$ resuelve la ecuación BS en log-precio:

\footnotetext{
${ }^{7}$ En inglés "boundary conditions $(B C)$ "
} 


$$
\begin{array}{cc}
\partial_{\tau} v-A^{B S-\log } v+r v=0 & \text { en } \mathbb{R} \times(0, T \\
v(0, x)=f\left(e^{x}\right) & \text { en } \quad \mathbb{R} \times(0, T)
\end{array}
$$

La ecuación de BS (4) es una ecuación diferencial 'parabólica' que puede perder, sin embargo, este carácter si la volatilidad $\sigma$ es pequeña, lo cual causa problemas a la hora de su solución numérica, por lo tanto y también para la derivación de una solución en forma cerrada, es importante darse cuenta de que (4) mediante las adecuadas transformaciones, es equivalente a la ecuación del calor,

$$
\frac{\partial u}{\partial \tau}-\frac{\partial^{2} u}{\partial x^{2}}=0
$$

que es también una ecuación parcial diferencial parabólica y describe un proceso de difusión. Esto es otra muestra más de la relación entre física y finanzas, de hecho, hay multitud de demostraciones y derivaciones de cómo llegar desde la ecuación del calor hasta la ecuación de BS.

Finalmente, para configurar los métodos en diferencias finitas en un dominio computacional acotado, debemos truncar la ecuación BS a un dominio de este tipo: dado $R>0$, restringimos (9) a $|x|<R$. Esta restricción significa que necesitamos condiciones de contorno artificiales en $x= \pm R$, siendo las condiciones más simples las homogéneas (o barrera), donde se denota por $v^{R}(x, t)$ la solución al problema truncado

$$
\begin{aligned}
& \partial_{\tau} v^{R}-A^{B S-\log } v^{R}+r v^{R}=0 \quad \text { en }(-R, R) \times(0, T), \\
& v^{R}(0, x)=f\left(e^{x}\right) \quad|x|<R \\
& v^{R}( \pm R, t)=0 \quad 0<t<T
\end{aligned}
$$

\section{PLANTEAMIENTO DEL PROBLEMA: DIFERENCIAS FINITAS PARA UNA OPCIÓN PUT EUROPEA.}

Consideremos la ecuación BS analizada anteriormente

$$
\begin{gathered}
\frac{\partial V}{\partial t}+\frac{\sigma^{2}}{2} S^{2} \frac{\partial^{2} V}{\partial S^{2}}+r S \frac{\partial V}{\partial S}-r V=0 \quad \text { en } \mathbb{R}_{+} \times J \\
V(S, T)=h(S) \text { en } \mathbb{R}_{+},
\end{gathered}
$$

donde $\mathrm{J}:=[0, \mathrm{~T}]$. Dejando que $u(x, \tau)=\mathrm{V}\left(e^{x}, T-t\right)$, entonces u resuelve que

$$
\begin{gathered}
\frac{\partial u}{\partial \tau}+\frac{\sigma^{2}}{2} \frac{\partial^{2} u}{\partial x^{2}}+\left(\frac{\sigma^{2}}{2}-r\right) \frac{\partial u}{\partial x}-r u=0 \quad \text { en } \mathbb{R} \times J \\
u(x, 0)=\psi(x) \quad \text { en } \mathbb{R},
\end{gathered}
$$

donde $\psi(x):=h\left(e^{x}\right)$. Desde que no podemos discretizar las ecuaciones (10) y (11), debemos truncarlas en el dominio acotado [a,b] e imponer condiciones de contorno

$$
\begin{gathered}
\frac{\partial u}{\partial \tau}+\frac{\sigma^{2}}{2} \frac{\partial^{2} u}{\partial x^{2}}+\left(\frac{\sigma^{2}}{2}-r\right) \frac{\partial u}{\partial x}-r u=0 \quad \text { en }[a, b] \times J \\
u(x, 0)=\psi(x) \quad \text { en }[a, b] \\
u(a, \tau)=g_{a}^{D}(\tau), u(b, \tau)=g_{b}^{D}(\tau) \quad \text { en } J \\
\text { obien } \frac{\partial u}{\partial x}(a, \tau)=g_{a}^{N}(\tau), \frac{\partial u}{\partial x}(b, \tau)=g_{b}^{N}(\tau) \quad \text { en } J \\
\text { obien } \frac{\partial u}{\partial x}(a, \tau)=g_{a}^{N}(\tau), u(b, \tau)=g_{b}^{D}(\tau) \text { en } J
\end{gathered}
$$




$$
\text { o bien } \quad u(a, \tau)=g_{a}^{D}(\tau), \frac{\partial u}{\partial x}(b, \tau)=g_{b}^{N}(\tau) \text { en } J
$$

Discretizamos (12)-(14) mediante diferencias finitas en $x \in[a, b]$ con una malla o red de tamaño $h=\frac{b-a}{N+1}$ y mediante el esquema- $\theta$ en $\tau \in[0, T]$ con M intervalos de tiempo de tamaño $k=\frac{T}{M}$. Por otro lado, si dejamos que $u_{i}^{m}:=u\left(x_{i}, \tau_{m}\right)$ y que $\underline{u}^{m}:=\left(u_{1}^{m}, \ldots, u_{N}^{m}\right)^{\mathrm{T}}$. Finalmente, obtenemos un sistema lineal para resolver en cada intervalo de tiempo del tipo

$$
\mathbf{B} \underline{u}^{m+1}=\mathbf{C} \underline{u}^{m}+\underline{b}^{m}, \quad m=0, \ldots, M-1
$$

Por lo tanto, lo que nos proponemos, una vez planteado el problema, es investigar la precisión/comportamiento de la solución numérica bajo diferentes condiciones de contorno y/o diferentes discretizaciones de los parámetros $k, h, \theta$.

\section{APLICACIÓN DE MÉTODOS NUMÉRICOS PARA SOLUCIÓN ÓPTIMA: PRECISIÓN Y COMPORTAMIENTO.}

\subsection{Demostración de la inestabilidad del esquema- $\theta$}

En primer lugar, desde un análisis de estabilidad, sabemos que el esquema- $\theta$ es estable si el factor de amplificación

$$
r(\mu k):=\frac{1+(1-\theta) \mu k}{1-\theta \mu k}
$$

satisface $|r(\mu k)| \leq 1$. Aquí, $k$ indica el intervalo de tiempo y $\mu=-\lambda_{i, N}, 1 \leq i \leq N$, con $\lambda_{i, N}$ como auto-valores (o valores propios) de la matriz $\frac{1}{k}$ (B-C) $\in \mathbb{R}^{N \times N}$. Asumiendo que $0<\lambda_{1, N} \leq \lambda_{2, N} \leq \cdots \leq$ $\lambda_{N, N}$, podemos demostrar que el esquema- $\theta$ anterior es incondicionalmente estable si $\frac{1}{2} \leq \theta \leq 1$ y que el intervalo de tiempo $k$ debe satisfacer

$$
k \leq \frac{2}{(1-2 \theta) \lambda_{N, N}}
$$

si $0 \leq \theta<\frac{1}{2}$.

Para ello, tenemos que

$$
\begin{aligned}
\left|r\left(\lambda_{i, N} k\right)\right| \leq 1 & \Leftrightarrow\left|1-\frac{k \lambda_{i, N}}{1+\theta k \lambda_{i, N}}\right| \leq 1 \\
& \Leftrightarrow 0 \leq \frac{k}{\frac{1}{\lambda_{i, N}}+\theta k} \leq 2 \\
& \Leftrightarrow 0 \leq k \leq \frac{2}{\lambda_{i, N}}+2 \theta k
\end{aligned}
$$

Ahora, si $0 \leq \theta<\frac{1}{2}$, entonces $0 \leq k(1-2 \theta)$ y así la última condición puede ser reformulada como

$$
0 \leq k \leq \frac{2}{\lambda_{i, N}}+2 \theta k \stackrel{\theta<\frac{1}{2}}{\Longleftrightarrow} 0 \leq k(1-2 \theta) \leq \frac{2}{\lambda_{i, N}} .
$$

Si $\frac{1}{2} \leq \theta \leq 1$, entonces $2 k \theta \geq k$ y así, la condición $0 \leq k \leq \frac{2}{\lambda_{i, N}}+2 \theta k$ siempre se mantiene, independientemente de $\lambda_{i, N}>0$. 


\subsection{Cálculo de $\lambda_{N, N}$ en caso de condiciones de contorno límite}

En el caso de condiciones de contorno límite, podemos encontrar una expresión para $\lambda_{N, N}$, para ello necesitamos la matriz $\frac{1}{k}$ (B-C). De este modo, usando diferencias finitas de segundo orden en espacio (es decir, $\frac{1}{h^{2}}\left(u_{i+1}^{m}-2 u_{i}^{m}+u_{i-1}^{m}\right)$ para $\partial_{x x} u(x, \tau)$ y $\frac{1}{2 h}\left(u_{i+1}^{m}-u_{i-1}^{m}\right)$ para $\partial_{x} u(x, \tau)$ ) el esquema- $\theta$ en el tiempo hasta la madurez $\tau$, tenemos:

$$
\frac{1}{k}\left(\underline{u}^{m+1}-\underline{u}^{m}\right)=\mathbf{A}_{h}\left(\theta \underline{u}^{m+1}+(1-\theta) \underline{u}^{m}\right)
$$

$\operatorname{con} \mathbf{A}_{h}=\mathbf{A}_{h}^{B S-} \quad-r \mathbf{I} \mathbf{y}$

$$
\left(\mathbf{A}_{h}^{B S-\log } \underline{u}\right)_{i}=\frac{\sigma^{2}}{2 h^{2}}\left(u_{i+1}-2 u_{i}+u_{i-1}\right)+\left(r-\frac{\sigma^{2}}{2}\right) \frac{1}{2 h}\left(u_{i+1}-u_{i-1}\right) .
$$

Entonces, la matriz es tri-diagonal $\left(\mathbf{A}_{h}=\operatorname{diag}\left(\alpha^{-}, \beta, \alpha^{+}\right)\right)$con cuyas entradas vienen dadas por

$$
\alpha^{ \pm}=\frac{\sigma^{2}}{2 h^{2}} \pm \frac{1}{2 h}\left(r-\frac{\sigma^{2}}{2}\right), \quad \beta=-\frac{\sigma^{2}}{2}-r .
$$

De (16) sigue (en el caso de condiciones de contorno cero)

$$
\underbrace{\left(\mathbf{I}-\theta k \mathbf{A}_{h}\right)}_{=: \mathbf{B}} \underline{u}^{m+1}=\underbrace{\left(\mathbf{I}+(\mathbf{1}-\theta) k \mathbf{A}_{h}\right)}_{=: \mathbf{C}} \underline{u}^{m}
$$

y así, de este modo $\frac{1}{k}(\mathbf{B}-\mathbf{C})=-\mathrm{A}_{h}=\operatorname{diag}\left(-\alpha^{-},-\beta,-\alpha^{+}\right) \in \mathbb{R}^{N \times N}$ según el método de convergencia de diferencias finitas, hallamos los auto-valores $\lambda_{i, N}, i=1, \ldots, N \mathrm{y}$

$$
\lambda_{N, N}=\frac{\sigma^{2}}{h^{2}}+r-\frac{1}{h} \sqrt{\frac{\sigma^{4}}{h^{2}}-\left(r-\frac{\sigma^{2}}{2}\right)^{2}} \cos \frac{N \pi}{N+1}
$$

Para $h$ lo suficientemente pequeño, una buena aproximación a $\lambda_{N, N}$ es $\lambda_{N, N} \approx 2 \frac{\sigma^{2}}{h^{2}}+r \approx 2 \frac{\sigma^{2}}{h^{2}}$.

\subsection{Hipótesis $g_{a}^{D}(\tau)=g_{a}^{D}(\tau)=0$}

\subsubsection{Aproximación para $\theta=0$ (Esquema Euler explícito)}

El siguiente paso en nuestro propósito de comprobar el comportamiento y la precisión de nuestra solución numérica es la realización de simulaciones o tests para los valores de $\theta$ tanto cuando la condición (15) es satisfecha como cuando no lo es y compararemos la solución numérica con la fórmula de Black-Scholes mediante el gráfico del error nodal absoluto.

Si $\theta=0$, el intervalo de tiempo $k$ debe satisfacer $k \leq \frac{2}{\lambda_{N, N}} \approx \frac{h^{2}}{\sigma^{2}}$.

Tomamos $T=1, K=20, \sigma=0.3, r=0.05,[a, b]=[-5,5]$ y $N=500$ puntos en la cuadrícula para el precio-log de $x$. De acuerdo con la aproximación $k \leq \frac{h^{2}}{\sigma^{2}}$, tendríamos que coger $M \approx 226$ intervalos de tiempo para cumplir el criterio de estabilidad. Dicha comparación y resultado se puede apreciar en la (figura 1) 

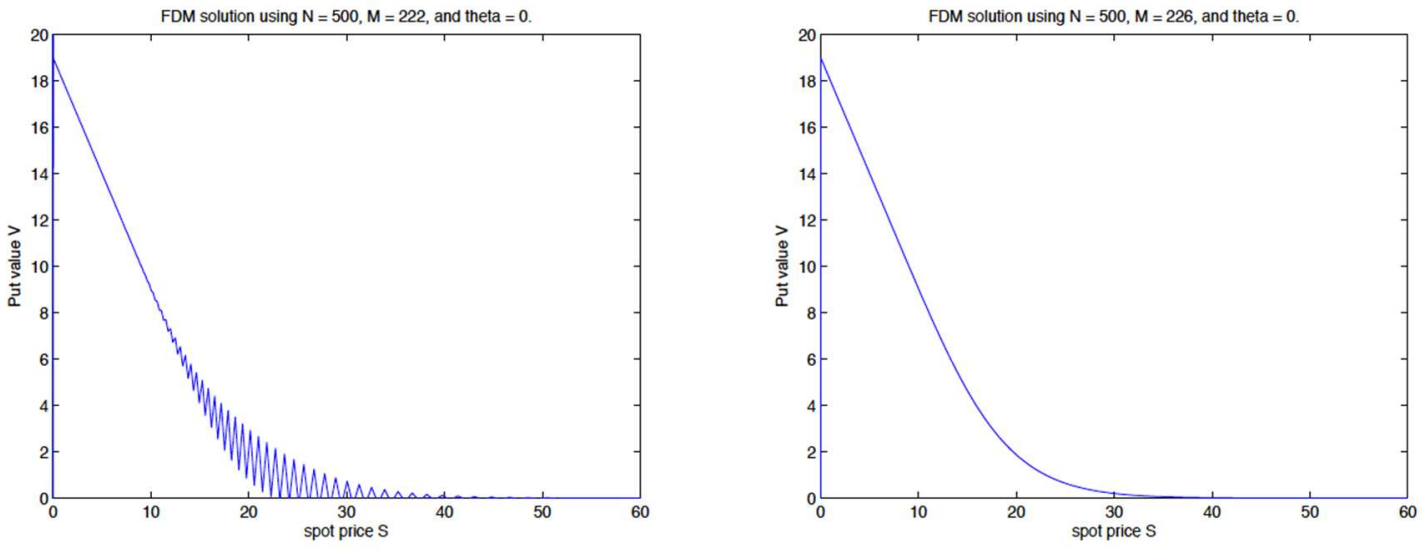

Figura 1: izquierda: criterio de estabilidad $k \leq \frac{2}{\lambda_{N, N}}$ no satisfecho. Derecha: criterio de estabilidad satisfecho.

4.3.2. Aproximación para $k=h$ y esquema Euler implícito $\theta=1$ y $\theta=\frac{1}{2}$ (Crank-Nicholson).

Si $k=h$, podemos esperar que el error sea de orden $\mathcal{O}(h)$ si $\theta=1$, y $\mathcal{O}\left(h^{2}\right)$ si $\theta=\frac{1}{2}$. Así, de este modo, podemos esperar un error más pequeño para $\theta=\frac{1}{2}$. Numéricamente, esto se confirma cuando seleccionamos, en nuestra simulación los mismos valores que en el caso del punto 4.1, con $N=999$ y así, $M=\frac{T(N+1)}{b-a}=100$ intervalos de tiempo (ver figura 3).
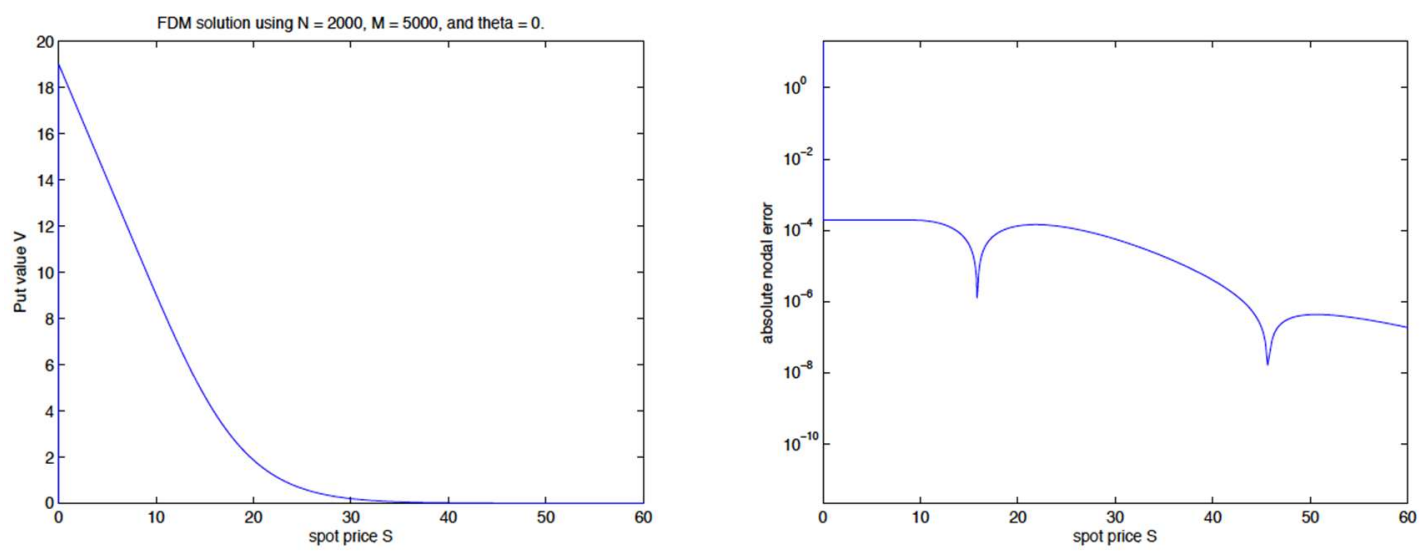

Figura 2: solución numérica y error nodal absoluto para $N=2000, M=5000$. Mala aproximación en $S \approx 0$ debido a las condiciones de contorno cero.

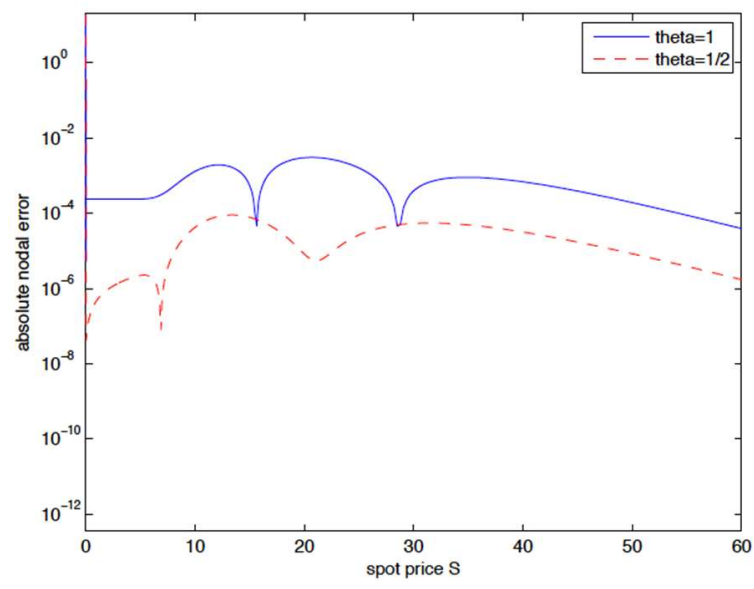

Figura 3: El error nodal absoluto es entre 10-100 veces más pequeño si usamos $\theta=\frac{1}{2}$. 


\subsection{Hipótesis $g_{a}^{D}(\tau)=K e^{-r \tau}-e^{a}$ y $g_{b}^{D}(\tau)=0$}

Para la simulación de esta hipótesis seguiremos los mismos pasos que para la hipótesis anterior, de este modo:

\subsubsection{Aproximación para $\theta=0$ (Esquema Euler explícito)}

Como hemos establecido, si tomamos $g_{a}^{D}(\tau)=K e^{-r \tau}-e^{a}, g_{b}^{D}(\tau)=0$, podemos justificar que $g_{a}^{D}(\tau)$ es una elección apropiada y con unas condiciones de contorno razonables. Así, sabemos que $\left.V_{C}(S, t)\right|_{S=0}=0$ y $\left.V_{P}(S, t)\right|_{S \rightarrow \infty}=0$.

De la paridad put-call $S+V_{P}-V_{C}=K e^{-r(T-t)}$ concluimos que $V_{P}(S, t) \approx K e^{-r(T-t)}-S, S \rightarrow 0$. Así, $u(x, \tau) \approx K e^{-r \tau}-e^{x}, \quad x \rightarrow-\infty$.

Nótese que las matrices B y $\mathrm{C}$ son como en el caso inicial descrito al comienzo del punto 4. Para encontrar $\underline{b}^{m}$, consideraremos la primera ecuación en (17)

$$
\begin{aligned}
-\theta k \alpha^{-} u_{0}^{m+1}+ & (1-\theta k \beta) u_{1}^{m+1}-\theta k \alpha^{+} u_{2}^{m+1} \\
= & (1-\theta) k \alpha^{-} u_{0}^{m}+(1+(1-\theta) k \beta) u_{1}^{m}+(1-\theta) k \alpha^{+} u_{2}^{m}
\end{aligned}
$$

Los valores $u_{0}^{m+1}, u_{0}^{m}$ son dados por $u_{0}^{m+1}=g_{a}^{D}\left(\tau_{m+1}\right)$ y $u_{0}^{m}=g_{a}^{D}\left(\tau_{m}\right)$ respectivamente. Así,

$$
\begin{gathered}
(1-\theta k \beta) u_{1}^{m+1}-\theta k \alpha^{+} u_{2}^{m} \\
=(1+(1-\theta) k \beta) u_{1}^{m}+(1-\theta) k \alpha^{+} u_{2}^{m}+\underbrace{\theta k \alpha^{-} g_{a}^{D}\left(\tau_{m+1}\right)+(1-\theta) k \alpha^{-} g_{a}^{D}\left(\tau_{m}\right)}_{=\left(\underline{b}^{m}\right)_{1}} .
\end{gathered}
$$

De lo cual sigue que $\left(\underline{b}^{m}\right)_{i}=0, \quad i=2, \ldots, N, \mathrm{y}$

$$
\left(\underline{b}^{m}\right)_{1}=k \alpha^{-}\left(\theta\left(K e^{-r \tau_{m+1}}-e^{a}\right)+(1-\theta)\left(K e^{-r \tau_{m}}-e^{a}\right)\right) .
$$

En este caso de esta hipótesis y procediendo de forma similar que en el punto 4.3, es decir, simulando $\operatorname{con} \theta=0$ y recordemos, satisfaciendo la condición (15), la capa límite $S \approx 0$ es eliminada.
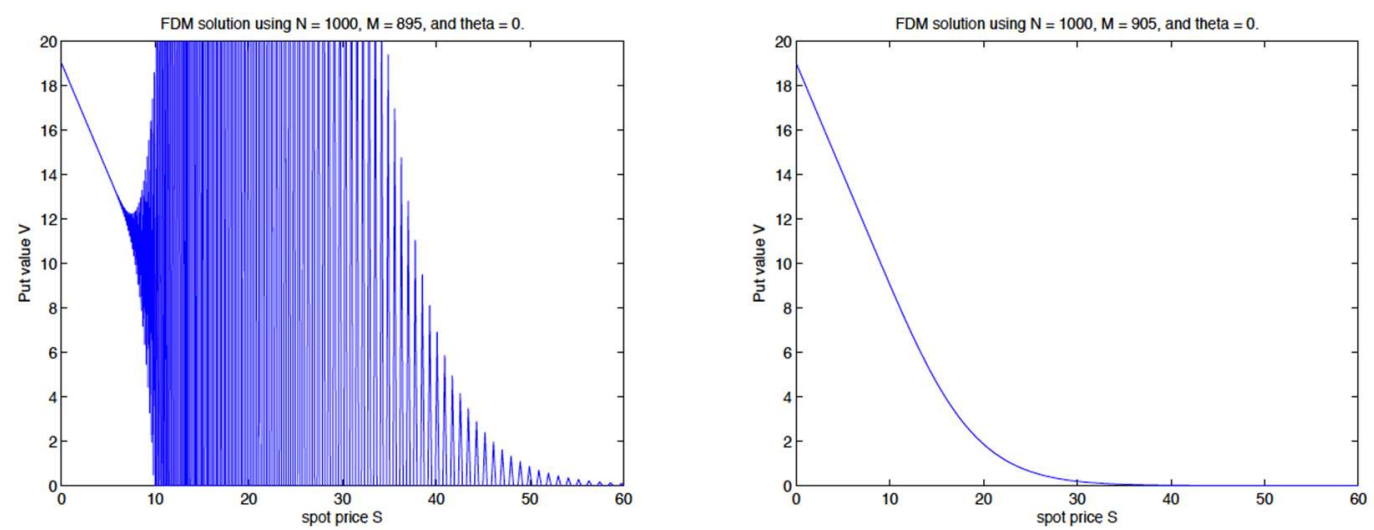

Figura 4: El error nodal absoluto es entre 10-100 veces más pequeño si usamos $\theta=\frac{1}{2}$.

4.4.2. Aproximación para $k=h$ y esquema Euler implicito $\theta=1$ y $\theta=\frac{1}{2}$ (Crank-Nicholson).

Tomando los mismos valores que en el apartado 4.3.2., vemos en la figura 5 que la aproximación en $S \approx 0$ es bastante buena. 


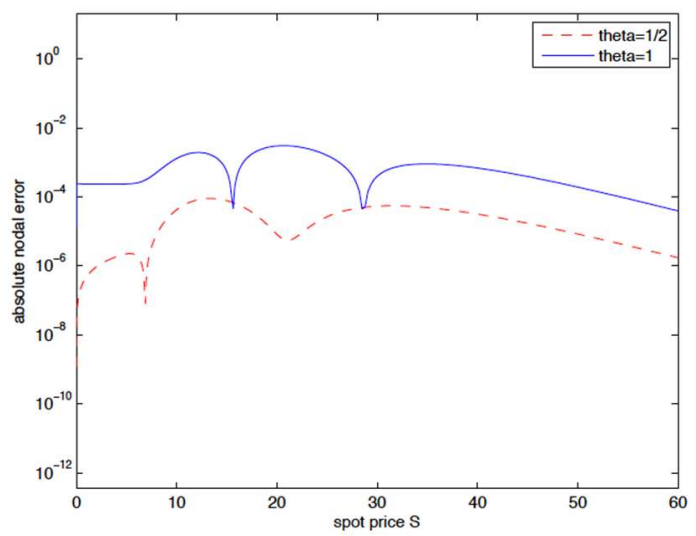

Figura 5: Comparativa del error nodal absoluto para $\theta=0$ y $\theta=\frac{1}{2}$.

\subsection{Solución numérica mediante la aproximación $\frac{1}{2 h}(-3 u(a, \tau)+4 u(a+h, \tau)-u(a+2 h, \tau))$}

Dejemos que $u(x, \cdot) \in C^{3}$. Usando la expansión de Taylor en torno $x=a$ resulta

$$
\begin{gathered}
u(a+h, \tau)=u(a, \tau)+h \partial_{x} u(a, \tau)+\frac{1}{2} h^{2} \partial_{x x} u(a, \tau)+O\left(h^{3}\right) \\
u(a+2 h, \tau)=u(a, \tau)+2 h \partial_{x} u(a, \tau)+2 h^{2} \partial_{x x} u(a, \tau)+O\left(h^{3}\right)
\end{gathered}
$$

Multiplicando la primera ecuación por 4 y restando la segunda ecuación elimina la segunda derivada, así tendremos

$$
\partial_{x} u(a+\tau)=\frac{1}{2 h}(-3 u(a, \tau)+4 u(a+h, \tau)-u(a+2 h, \tau))+O\left(h^{2}\right) .
$$

Lo anterior implica que usemos

$$
\frac{1}{2 h}\left(-3 u_{0}^{m}+4 u_{1}^{m}-u_{2}^{m}\right)=g_{a}^{N}\left(\tau_{m}\right) \Rightarrow \frac{1}{3}\left(4 u_{1}^{m}-u_{2}^{m}-2 h g_{a}^{N}\left(\tau_{m}\right)\right)
$$

para incorporar condiciones de contorno Neumann.

De nuevo, para encontrar las matrices $\mathbf{B}, \mathbf{C}$ y los vectores $\underline{b}^{m}$, consideramos la ecuación (18); en ella reemplazamos $u_{0}^{m}$ por la expresión anterior (y de forma similar para $u_{0}^{m+1}$ ), donde después de agrupar los términos apropiados tenemos

$$
\begin{aligned}
\left(-\frac{4}{3} \theta k \alpha^{-}+1\right. & +\theta k \beta) u_{1}^{m+1}+\left(\frac{1}{3} \theta k \alpha^{-}-\theta k \alpha^{+}\right) u_{2}^{m+1} \\
& =\left(\frac{4}{3}(1-\theta) k \alpha^{-}+1+(1-\theta) k \beta\right) u_{1}^{m}+\left(-\frac{1}{3}(1-\theta) k \alpha^{-}+(1-\theta) k \alpha^{+}\right) u_{2}^{m} \\
& -\frac{2}{3} k h \alpha^{-}\left(\theta g_{a}^{N}\left(\tau_{m+1}\right)+(1-\theta) g_{a}^{N}\left(\tau_{m}\right)\right),
\end{aligned}
$$

tal que

$$
\underbrace{\left(\mathbf{I}-\theta k \widetilde{\mathbf{A}_{h}}\right)}_{=: \mathbf{B}} \underline{u}^{m+1}=\underbrace{\left(\mathbf{I}+(1-\theta) k \widetilde{\mathbf{A}_{h}}\right)}_{=: \mathbf{C}} \underline{u}^{m}+\underline{b}^{m}
$$

donde

$$
\left(\widetilde{A_{h}}\right)_{11}=\frac{4}{3} \alpha^{-}+\beta, \quad\left(\widetilde{A_{h}}\right)_{12}=-\frac{1}{3} \alpha^{-}+\alpha^{+}, \quad\left(\widetilde{A_{h}}\right)_{i j}=\left(\widetilde{A_{h}}\right)_{i j^{\prime}}
$$

y finalmente

$$
\left(\underline{b}^{m}\right)_{1}=\frac{2}{3} k h \alpha^{-} e^{a}, \quad\left(\underline{b}^{m}\right)_{i}=0, \quad i=2, \ldots, N
$$


Simulando con los mismos valores que en el punto 4.3.1 y N=5000, $\mathrm{M}=1000$, vemos (figura 6) que el error nodal absoluto es menor que 10-6.

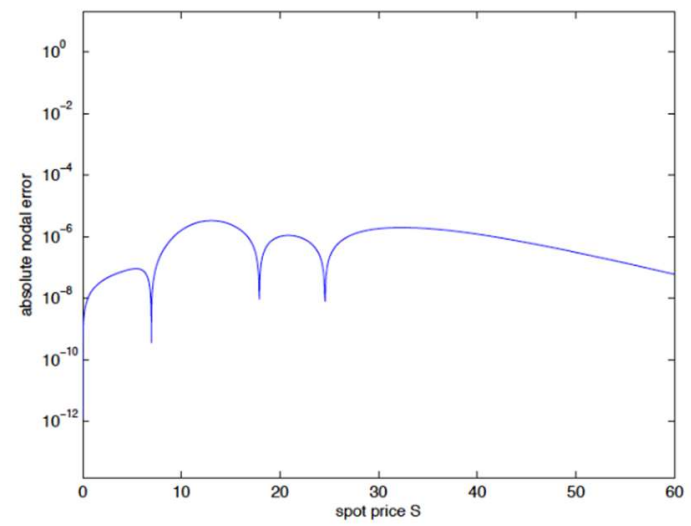

Figura 6: Error nodal absoluto para $N=5000, M=1000$

Finalmente, terminamos la sección con una observación importante y es que, en el modelo de BlackScholes, las ganancias, distribuidas de forma gaussiana, son generadas por la ecuación diferencial estocástica

$$
d x=\left(r-\frac{\sigma^{2}}{2}\right) d t+\sigma d B
$$

donde $\sigma$ es constante. La correspondiente ecuación de Fokker-Planck es

$$
\frac{\partial f}{\partial t}=-\left(r-\frac{\sigma^{2}}{2}\right) \frac{\partial f}{\partial x}+\frac{\sigma^{2}}{2} \frac{\partial^{2} f}{\partial x^{2}}
$$

Por lo tanto, la distribución del precio log-normal es descrita por

$$
d p=r p d t+\sigma p d B
$$

y la distribución log-normal es función Green para

$$
\frac{\partial g}{\partial t}=-r \frac{\partial}{\partial p}(p g)+\frac{\sigma^{2}}{2} \frac{\partial^{2}}{\partial p^{2}}\left(p^{2} g\right)
$$

donde $g(p, t) d p=f(x, t) d x$ o $f(x, t)=p g(p, t) \operatorname{con} x=\ln p$ (McCauley, 2004 y Watanabe, 2014).

\section{CONCLUSIONES}

Al comienzo hemos enumerado algunos elementos y teorías de la física y la mecánica cuántica que tienen su aplicación al campo de la economía y más concretamente, de las finanzas.

En el caso que hemos desarrollado hemos deducido que la ecuación Fokker-Planck es la base sobre la que se asienta el proceso estocástico en el que se basa el modelo de Black-Scholes, además, se ha presentado una caracterización probabilística del precio justo de la ecuación diferencial estocástica, se ha calculado vía solución numérica siguiendo el método de diferencias finitas y hemos comprobado la precisión y el comportamiento bajo diferentes condiciones de contorno y con diferentes valores de los parámetros de discretización $k, h, \theta$.

De este modo, podemos concluir que la ecuación de Fokker-Planck se configura como uno de los métodos más óptimos para resolver cualquier ecuación diferencial estocástica, ya que describe no solo las propiedades de estacionariedad, sino que también describe el comportamiento dinámico del proceso estocástico en cuestión, siendo aplicable tanto a sistemas en equilibrio como a sistemas en situación de no equilibrio.

Dentro de la línea de trabajo, orientada tanto a la aplicación de modelos y teorías del campo de la física a la economía financiera como del uso de métodos numéricos para la resolución de estos 
problemas, se podrían aplicar conceptos de la mecánica cuántica a la modelización del mercado de valores, en línea con autores como Zhang (Zhang y Huang, 2010) o tratar de modelizar el rendimiento de ciertos activos, de forma similar a McCauley y Gunaratne (2000) que muestran cómo la formulación de fluctuaciones de Fokker-Planck puede ser utilizada con una volatilidad local (coeficiente de difusión) para generar una distribución exponencial para rendimientos de estos activos.

A pesar de las diferencias que puedan surgir entre físicos y economistas, revisando la literatura, es innegable la contribución que se ha hecho en los últimos años debido a este nuevo campo y el futuro presenta numerosas oportunidades y retos de investigación en ambas áreas como para no establecer lazos de unión y colaboración entre ambas partes.

\section{REFERENCIAS BIBLIOGRÁFICAS}

BAAQUIE, B. E. (2013): "Financial Modeling and Quantum Mathematics" Computer Mathematics with Applications, No. 65, pp.1665-1673

BLACK, F. y SCHOLES, M. (1973): "The Pricing of Options and Corporate Liabilities" Journal of Political Economy, Vol. 81, No. 3 (May - Jun., 1973), pp. 637-654.

BORLAND, L., BOUCHAUD, J-P., MUZY, J-F. y ZUMBACH, G. (2006): "The Dynamics of Financial Markets Mandelbrot's Multifractal Cascades, and Beyond. (Consultado en línea el 02 de diciembre en: https://arxiv.org/abs/cond-mat/0501292).

BRANDIMARTE, P. (2002). Numerical methods in finance. A MATLAB-Based introduction. New York: Ed. John Wiley \& Sons.

CHEN, M. J. (2018): "Baryonic Beta Dynamics: An Econophysical Model of Sistematic Risk" Estudios de Economía Aplicada, Vol. 36-1, pp. 263-276.

CLARA RAHOLA, J.; PUERTAS, A. M.; SÁNCHEZ GRANERO, M. A.; TRINIDAD SEGOVIA, J. E. y DE LAS NIEVES, F. J.: "Diffusive and Arrestedlike Dynamics in Currency Exchange Markets". Physical Review Letters, Vol.118, No. 6 (Feb., 2017). Consultado en línea el 10 de enero: https://journals.aps.org/prl/abstract/10.1103/PhysRevLett.118.068301.

COMITÉ, Premio Nobel, (2001): "George A. Akerlof, A. Michael Spence, Joseph E. Stiglitz: Markets with Asymmetric Information," Premio Nobel de Economía. Documentos 2001-2.

COURANT, R.; FRIEDRICHS, K. y LEWY, H., (1928): "On The Partial Difference Equations of Mathematical Physics", Matematische Annalen No. 100, pp. 32-74

DESLOGE, EDWARD A. (1963): "Fokker-Planck Equation” American Journal of Physics, Vol. 31, No. 4, pág. 237.

EDGAR, P. E. (1994): Fractal Market Analysis: Applying Chaos Theory to Investment and Economics. Nueva York, Ed. John Wiley and Sons.

GEORGESCU-ROEGEN, N. (1971): The Entropy Law and the Economic Process. Cambridge, Massachusetts: Ed. Harvard University Press.

GINGRAS, Y. y SCHINCKUS, C. (2012): "The institutionalization of econophysics in the shadow of physics" J. Hist. Econ. 34 pp. 109-130.

HERZOG, B. (2015): “An Econophysics Model of Financial Bubbles”, Natural Science. No. 7, pp. 55-63. Publicado on-line con licencia Creative Commons. (Consultado en noviembre 2018 en https://www.scirp.org/journal/ns/).

ILINSKI, K. (2001): Physics of Finance: Gauge Modelling in Non-Equilibrium Pricing. Nueva York, Ed. John Wiley \& Sons.

INGBER, L. (1990): "Statistical-mechanical Aids to Calculating Term-Structure Models" www.ingber.com, fecha de consulta: diciembre 2018.

JOVANOVIC, F. y SCHINCKUS, C. (2016): "Breaking Down the Barriers Between Econophysics and Financial Economics". International Review of Financial Analysis. Vol. 47, pp. 256-266, (octubre 2016).

JOVANOVIC, F. y SCHINCKUS, C (2017): Econophysics and Financial Economics: An Emerging Dialogue. Nueva York, Ed. Oxford University Press

KUTNER, R. y GRECH, D (2008): "Report on Foundation and Organization of Econophysics Graduate Courses at Faculty of Physics of University of Warsaw and Department of Physics and Astronomy of the Wroclaw University. Proceedings of the 3rd Polish Symposium on Econo- and Sociophysics, Wroclaw 2007". Acta Physica Polonica A, Vol. 118.No. 3.

LIGOU, J. (1986): "The Bolzmann-Fokker-Planck Equation”. Transport Theory and Statistical Physics, Vol. 15, No. 6-7, pp. 985-1005.

MANDELBROT, B. (1997): La Geometría Fractal de la Naturaleza. (Barcelona). Ed. Tusquests Editores, S.A.

MANDELBROT, B. y HUDSON, R. L. (2006): Fractales y Finanzas. (Barcelona). Ed. Tusquests Editores, S.A

MANTEGNA, R. M. y STANLEY, E. (2000). An Introduction to Econophysics: Correlations and Complexity in Finance. Reino Unido: Ed. Cambridge University Press.

McCAULEY, J. L. (2004). Dynamics of Markets: Econophysics and Finance. Cambridge, Reino Unido: Ed. Cambridge University Press.

McCAULEY, J. L. y GUNARATNE, G. H. (2003): "An Empirical Model of Volatility of Returns and Option Pricing". Physica A, Vol. 329, pp. 178-198.

PAVARD, B. y DUGDALE, J. (2001): "An Introduction to Complexity in Social Science". Tutorial para el proyecto COSI (HPRN-CT-2000-00068, Complexity in Social Science). GRIC-IRIT, Toulouse. 
RISKEN, H. y FRANK, T. (1996): The Fokker-Planck Equation. Methods of Solutions and Applications. Berlin, Ed. Springer-Verlag.

ROEHNER, B. M. (2002): Patterns of Speculation: A Study in Observational Econophysics. Reino Unido, Ed. Cambridge University Press.

SCHINCKUS, C. (2010): "Is Econophysics a new discipline?" Physica A 389 pp. 3814-3821.

SCHADEN, M. (2002): "Quantum Finance" Physica A: Statistical Mechanics and Its Applications, 316, pp. 511-538.

STANLEY, H. E; AFANASYEV, V.; y OTROS (1996): "Anomalous fluctuations in the dynamics of complex systems: from DNA and physiology to econophysics", Physica A 224 pp. 302-321.

STEPHEN, S. y YAU, T. (2004): "Computation of Fokker-Planck Equation" Quarterly of Applied Mathematics, Vol. LXII, No. 4 (Diciembre, 2004), pp. 643-650.

VOIT, J. (2005): The Statistical Mechanics of Financial Markets (3era edición). Berlin, Ed. Springer-Verlag.

WATANABE, K. (2014): Integral Transform Techniques for Green's Function. Nueva York, Ed. Springer-Verlag.

WATTS, D. J. (2004): Six Degrees: The Science of a Connected Age. Nueva York. Ed.: W.W. Norton \& Company Ltd.

ZHANG, C. y HUANG, L. (2010): “A Quantum Model for the Stock Market”, Physica A. Vol. 389. No. 24, pp. 57695775 .

\section{ANEXO I: Código de simulación para la ecuación diferencial parcial de Black-Scholes en diferencias finitas mediante Matlab $\odot$}

Las soluciones obtenidas aplicando métodos numéricos han sido hechas con el software Matlab, cuyo script y rutina se adjuntan a continuación.

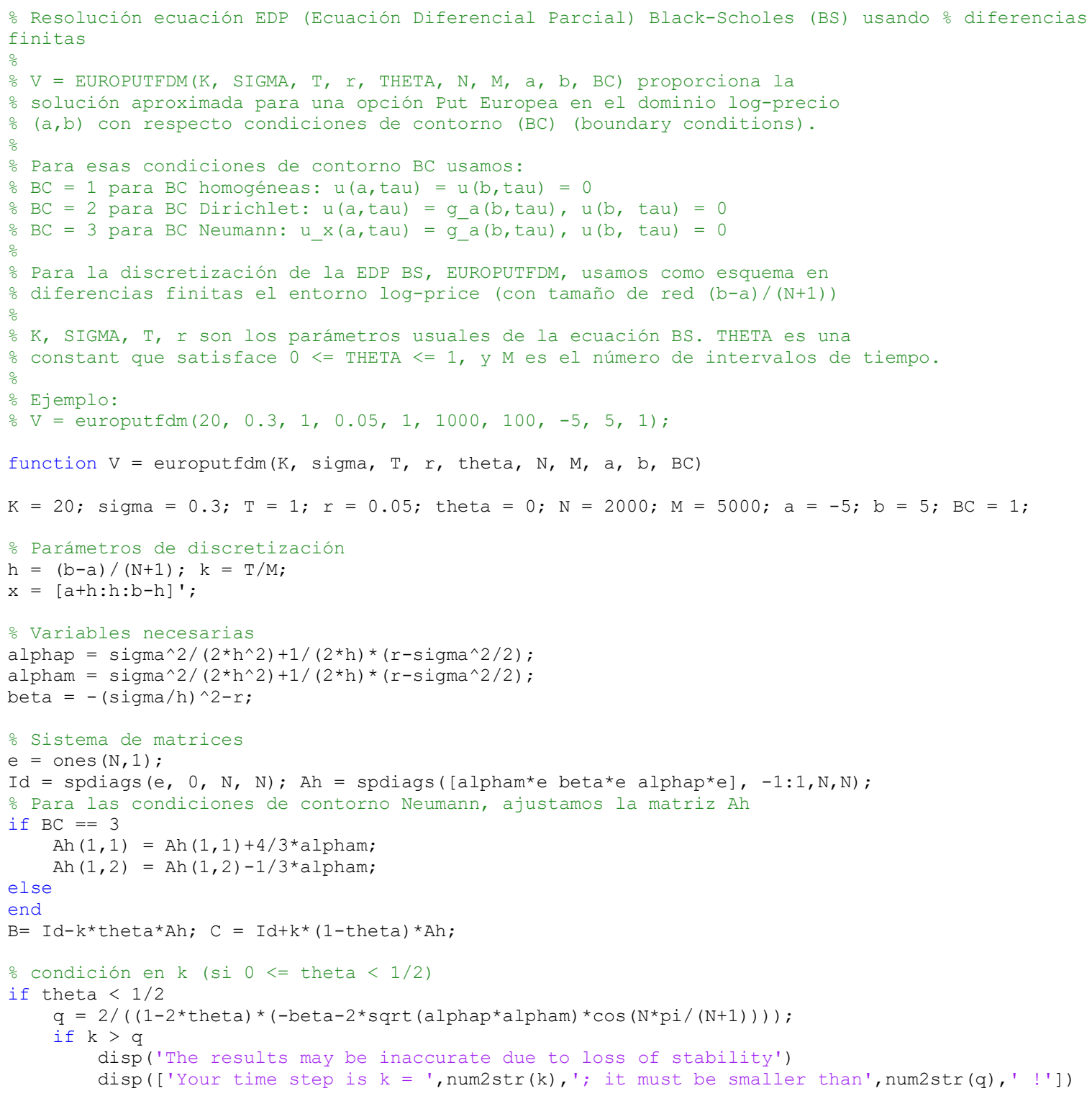




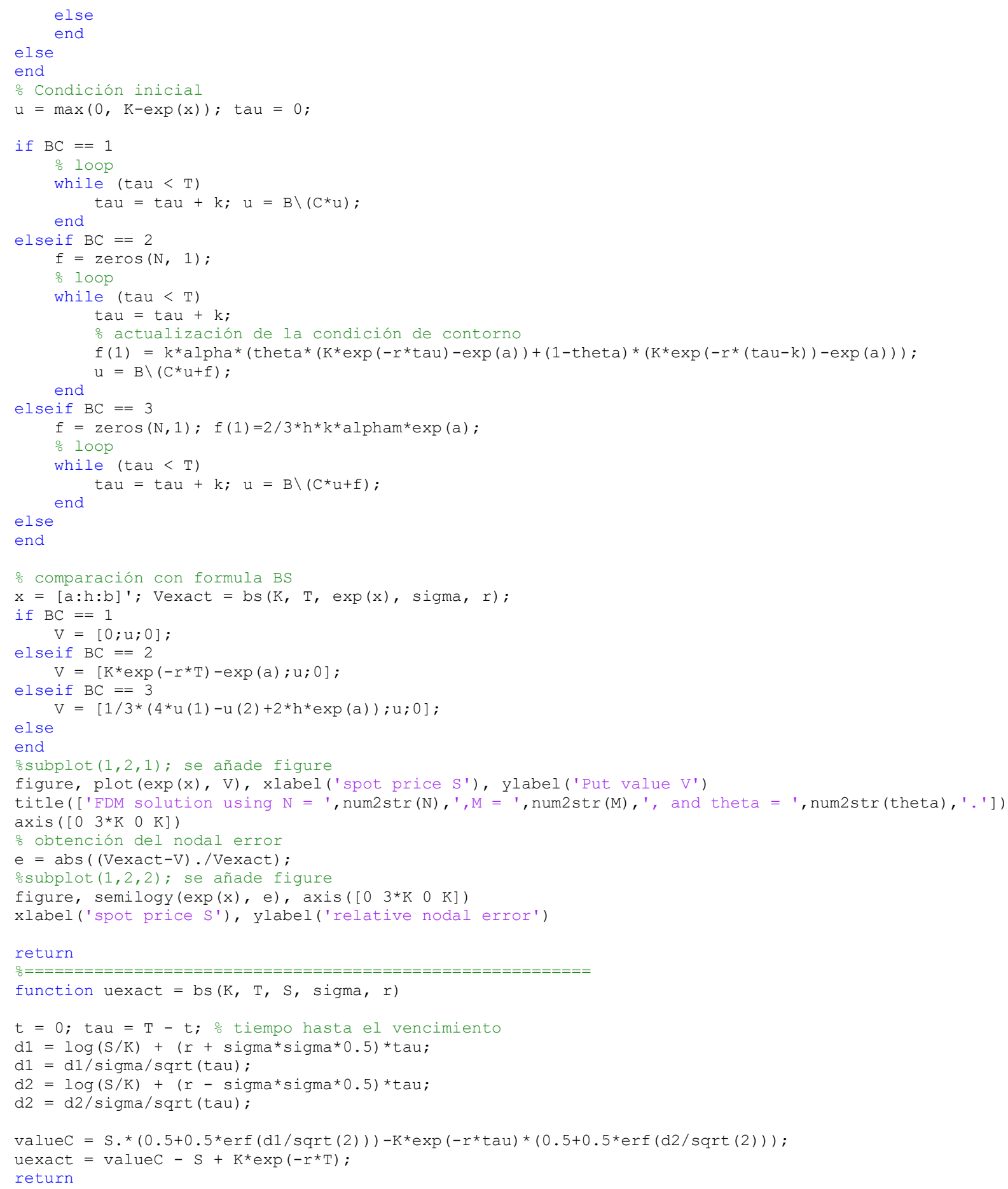

